\title{
Assessing the impact of mobile travel applications on tourism industries
}

\author{
Nehal Mohamed Abd Elzaher Eltayeb \\ Lecturer at Cairo Higher Institute for Tourism and Hotels \\ Tourism Department
}

\begin{abstract}
Smart phones and tablets have played an important role in our lives as many of the mobile users spend their time using mobile applications. As the relationship between the tourism industry and technology grows, Smartphones continue make it easier to carry all the resources you need along your travels. Mobile users prefer comparing mobile travel applications to browsing the web or checking travel agencies 'web sites, especially while looking for hotels or flights. The main aims of this study are determining users' opinions regarding Mobile travel Applications. Identifying the relationship between demographic characteristics and customer loyalty and evaluating how customers view travel agencies websites. A survey was conducted to determine the views of a randomly selected sample of 228 of domestic tourists in Egypt and international tourists in relation to their mobile travel applications use. The results revealed that mobile travel users are increasing and the users' buying behavior show that they are loyal to mobile travel application.
\end{abstract}

Keywords Tourism, Mobile travel applications, Smartphone, tourists' loyalty and buying behavior

\section{Introduction}

"Mobile tourism" as an expression started to appear in the last two decades, and it involves using mobile devices as electronic tourist guides (Kenteris, et.al, 2009 ). The development of information technology is tremendous and this reflects on tourism field as well (Parro, 2013). Technological improvement and tourism have been going side by side for many years . Smartphone mobile applications are extremely important for the tourism industry as mobile applications have a considerable effect on tourism. They allow the user to access the contents through an installed application on the mobile devices (Alshattnawi, 2013). These mobile travel applications enable 
users to be more aware of their surroundings as tourists can look up information about a point of interest (POI) on their mobile (Alshattnawi, 2013).

"The smart tourism is explained as a holistic approach that provide tour information, service related to travel, such as destination, food, transportation, reservation, travel guide, conveniently to tourists through IT devices" (Chulmo et al., 2013).

This study aims to explain the importance of mobile travel applications in the tourism industry and to measure their impact on tourism to determine where tourists' ( users) position when it comes to adopting new technology ,in addition to measuring the loyalty of mobile travel applications users.

\section{The objectives are}

1- Assessing mobile travel applications effect on tourists' buying behavior

2- Evaluating users' loyalty to travel mobile applications .

3- Evaluating respondents' reviews of travel agencies websites, according to their experience.

\section{Literature review}

\section{Smart phones:}

Buhalis and costa (2006) stated that smart phones are wearable computing systems that can be used anywhere the person goes. "Smart phones are Mobile phones that combine communication with sophisticated personal use technology such as access to the Internet and the ability to download applications. The latest generation was introduced by Apple, in the form of its popular iPhone, in June 2007. Since then, a whole range of smart phones from competing brands using this technology have followed" ( Tourism e kit ,2017). Smartphones gather all needed technologies for augmentation in one small device (Yovcheva et al., 2012).

Business insider (2013) mentioned that Smart phones have created a new business opportunity in hotel booking, as they facilitate last minute booking .Furthermore, business travelers prefer to book their travels on mobile and consumers who use their mobile devices for travel-related services tend to have higher than-average incomes. Grieve (2010) research found that Smartphone users were more frequent travelers, heavier users of social networking media, and they used their Smartphone devices for a range of travel related activities, including information search, location and directional tools , and booking travels during their trips. 
Mamaghani, 2009 and Langelund, 2007 stated that "smart phones have improved the traveler experience and mobile travel will soon be a must have tool for travelers". Travel can no longer be seen as a completely separate entity from everyday life, as smartphones make it easier to use travel applications during their trips . (Mortiz, 2015)

\section{Mobile Travel Applications}

Jieun et al, (2014) defined mobile applications as "Software that provides information in various formats, according to the technical features of the mobile devices for which they are designed and which can provide information and specific support to their users, being dedicated to daily or business activities". Travel applications help to plan and arrange the whole trip on the mobile device, having the whole itinerary in one place. These mobile travel applications cover travel agencies, hotels, car rentals, airlines, travel information, weather forecasts, maps, etc. The World Travel Market Global Trends Report revealed that bookings made from mobile devices will reach $35 \%$ in 2018 (Wtm, 2017 ).

Furthermore, Mortiz (2015) shows that travel apps are still mostly used in the planning phase of arranging users' trips . Moreover, tourists use mobile applications on site as they enable users to select the best restaurants right on the spot, receive location based recommendations directly at the destination, or make use of GPS based navigation (Wang, Xiang and Fesenmaier, 2014).

Kenteris et al. (2007) divided existing commercial applications and research approaches in the field of mobile tourism into three main categories that involve :

- Tourist or museum guides with pre-installed applications that cannot be customized according to user preferences (rigidly defined content (in text, visual and auditory format)

- Mobile devices used to access mobile web portals to browse tourist information.

- Mobile electronic guides devices, which use either wireless or mobile network connections to access context awareness services.

Bicen and Sadikoglu' (2016) findings revealed that students plan their travels with mobile applications and social media content, choose their venues of interest using GPS, and, map applications, make price arrangements and compare hotels by using mobile applications. This indicates that they view mobile applications as reliable. 


\section{Buying Behavior and loyalty of Smartphone Users}

Yoo and Gretzel, 2012 stated that the availability of online applications are rapidly growing and changing communication, making decisions and socializing .This influences consumers' behavior and in turn tourism.

Dick and Basu (1994) mentions that customer loyalty is the strength of the relationship between an individual's relative attitude and repeat patronage. Fiedler (2013) revealed that customer loyalty towards Mobile Travel Guide Application have a positive attitude where only $(8 ; 16.3 \%)$ stated that they either disagree $(7 ; 14.3 \%)$ or strongly disagree $(1 ; 2.0 \%)$. Furthermore, Good work label (2015) published that $30 \%$ use mobile apps to find the best hotel deals , 29\% use mobile apps to find the best flight deals , $15 \%$ of users download travel apps to plan a trip ahead , 55.8\% use mobile apps to check weather, $49.1 \%$ use mobile apps to use mapping features , $62.1 \%$ use mobile apps to search nearby restaurant. $48.1 \%$ use mobile GPS to get travel directions , 46\% use apps to find hotels and $36.1 \%$ use mobile apps to look for popular places for shopping.

All of that shows that tourists can actually have a loyalty for mobile applications as they can make a difference in customers' attitude towards the applications that are being used for their trip arrangements.

\section{Hypothesis}

H1: Customer loyalty has a positive influence on buying behavior.

$\mathrm{H} 2$ : Usage of mobile travel applications has a positive influence on travel agencies website.

H3: There is a positive relationship between Demographic characteristics and customer loyalty.

\section{Methodology}

\subsection{Participants, instrument and measures}

This study uses the deductive approach and quantitative method to find out the causal relationships between studies independent and dependent variables, also a questionnaire was conducted to determine international and domestic travelers' opinions in relation to mobile travel applications. Only people who have smart phones were considered for this study. The questionnaire was randomly submitted to a sample of 228 national and international mobile travel application users. The research was started on the $6^{\text {th }}$ of June 2017 and ended on $10^{\text {th }}$ of February 2018. 


\subsection{Questionnaire lay out}

Respondents were asked to responded to a questionnaire, the first part dealt with respondents demographics, the second part emphasized tourists buying behavior of mobile travel applications, the third part identifies the usage of mobile travel applications, the fourth part evaluates the travel agencies' website offers, and the fifth part discusses the customer loyalty to travel mobile applications .This questionnaire was obtained from Fiedler, 2013, parro 2013.

\section{Results and discussion}

\subsection{Reliability and validity}

To verify how the survey measurements met the objectives of this study, A Cronbach's alpha was used. The lower limit for Cronbach's alpha is 0.70 (Pallant, 2005). The results of the reliability are shown in table 1. As the table shows Cronbach's alpha varies between 0.89-0.929 which are regarded as accepted reliability and the validity of the coefficient value above 0.35 which is very beneficial. Thus, the questionnaire items were considered reliable and valid

Table 1: Reliability and validity

\begin{tabular}{|l|c|c|}
\hline & validity & reliability \\
\hline Buying behavior & .466 & .865 \\
\hline Usage of mobile travel application & .464 & .928 \\
\hline Travel agencies website offers & .650 & .893 \\
\hline $\begin{array}{l}\text { Customer loyalty to travel mobile } \\
\text { applications }\end{array}$ & .589 & .897 \\
\hline
\end{tabular}

Table 2: Respondents' demographic profile

\begin{tabular}{|l|c|c|c|}
\hline Gender & Frequency & Percent & Valid Percent \\
\hline & 138 & 60.5 & 60.5 \\
\hline Male & 90 & 39.5 & 39.5 \\
\hline Female & 228 & 100.0 & 100.0 \\
\hline Total &
\end{tabular}

\begin{tabular}{|c|c|c|c|}
\hline Age & \multicolumn{3}{|l}{} \\
\hline $21-30$ & Frequency & Percent & Valid Percent \\
\hline $31-40$ & 86 & 37.7 & 37.7 \\
\hline $41-50$ & 102 & 44.7 & 44.7 \\
\hline $51-60$ & 32 & 14.0 & 14.0 \\
\hline Total & 8 & 3.5 & 3.5 \\
\hline Education & 228 & 100.0 & 100.0 \\
\hline
\end{tabular}


Assessing the impact of mobile travel applications on tourism industries

\begin{tabular}{|l|c|c|c|}
\hline & Frequency & Percent & Valid Percent \\
\hline Upper Secondary School/Vocational Education & 4 & 1.8 & 1.8 \\
\hline Bachelor's Degree & 112 & 49.1 & 49.1 \\
\hline Master's Degree & 48 & 21.1 & 21.1 \\
\hline Doctor's Degree & 64 & 28.1 & 28.1 \\
\hline Total & 228 & 100.0 & 100.0 \\
\hline
\end{tabular}

The result indicated that $60.5 \%$ of the respondents were males and $39.5 \%$ were females. Age wise the majority of respondents $(44.7 \%)$ were between $31-40,37.7$ are between $21-30,14 \%$ are between $41-50$ and only $3.5 \%$ are between 51-60. Regarding the education degree of respondents, the vast majority of respondents $(49.1 \%)$ had bachelor degrees , $(28.1 \%)$ have $\mathrm{PhDs}$ ,(21.1\%) have Masters degrees and only $1.8 \%$ are Upper Secondary School.

Table 3: Travelers' buying behavior

\begin{tabular}{|l|c|c|c|c|c|}
\hline \multicolumn{7}{|c|}{ Do you book hotels via mobile application? } \\
\hline & Frequency & Percent & Valid Percent & Mean & Std. Deviation \\
\hline Yes & 146 & 64.0 & 64.0 & 1.447 & .4983 \\
\hline No & 82 & 36.0 & 36.0 & & \\
\hline Total & 228 & 100.0 & 100.0 & & \\
\hline \multicolumn{7}{|c|}{ Do you book flights via mobile application? } \\
\hline Yes & Frequency & Percent & Valid Percent & Mean & Std. Deviation \\
\hline No & 126 & 55.3 & 55.3 & 1.360 & .4810 \\
\hline Total & 102 & 44.7 & 44.7 & & \\
\hline
\end{tabular}

\begin{tabular}{|c|c|c|c|c|c|}
\hline \multicolumn{6}{|c|}{ Where do you mostly book your flights? } \\
\hline & Frequency & Percent & $\begin{array}{c}\text { Valid } \\
\text { Percent }\end{array}$ & Mean & $\begin{array}{c}\text { Std. } \\
\text { Deviation }\end{array}$ \\
\hline $\begin{array}{l}\text { Online direct (on the airline's } \\
\text { website/mobile application }\end{array}$ & 70 & 30.7 & 35.1 & \multirow{6}{*}{2.061} & \multirow{6}{*}{1.0889} \\
\hline $\begin{array}{l}\text { Online indirect (through booking } \\
\text { websites/mobile application) }\end{array}$ & 86 & 37.7 & 37.7 & & \\
\hline $\begin{array}{l}\text { Offline direct (with the ticket office } \\
\text { of the airline) }\end{array}$ & 46 & 20.2 & 20.2 & & \\
\hline $\begin{array}{l}\text { Offline indirect eg.( through a } \\
\text { booking agency) }\end{array}$ & 10 & 4.4 & 4.4 & & \\
\hline I do not take flights & 16 & 7.0 & 7.0 & & \\
\hline Total & 228 & 100.0 & 100.0 & & \\
\hline
\end{tabular}




\begin{tabular}{|c|c|c|c|c|c|}
\hline \multicolumn{6}{|c|}{ Where do you mostly book your hotels? } \\
\hline & Frequency & Percent & $\begin{array}{c}\text { Valid } \\
\text { Percent }\end{array}$ & Mean & $\begin{array}{c}\text { Std. } \\
\text { Deviation } \\
\end{array}$ \\
\hline $\begin{array}{l}\text { Online direct (on the hotel's } \\
\text { website/mobile application) }\end{array}$ & 53 & 23.245 & 23.245 & \multirow{5}{*}{2.447} & \multirow{5}{*}{.9672} \\
\hline $\begin{array}{l}\text { Online indirect (through booking } \\
\text { websites/mobile application) }\end{array}$ & 143 & 62.71 & 62.71 & & \\
\hline $\begin{array}{l}\text { Offline indirect (e.g. through a } \\
\text { booking agency) }\end{array}$ & 12 & 5.26 & 5.26 & & \\
\hline I do not book hotels & 20 & 8.77 & 8.77 & & \\
\hline Total & 228 & 100.0 & 100.0 & & \\
\hline \multicolumn{6}{|c|}{ Which Mobile Travel Application have you used in booking a trip } \\
\hline & Frequency & Percent & $\begin{array}{c}\text { Valid } \\
\text { Percent }\end{array}$ & Mean & $\begin{array}{c}\text { Std. } \\
\text { Deviation }\end{array}$ \\
\hline $\begin{array}{l}\text { Directly from the airline, hotel, car } \\
\text { rental app (e.g. Egypt air, British } \\
\text { Airways, Hilton, Marriott, Hertz etc.) }\end{array}$ & 70 & 30.7 & 30.7 & \multirow{5}{*}{2.263} & \multirow{5}{*}{1.3476} \\
\hline $\begin{array}{l}\text { Tour operator apps (e.g. TUI, Thomas } \\
\text { Cook etc.) }\end{array}$ & 10 & 4.4 & 4.4 & & \\
\hline $\begin{array}{l}\text { Travel agencies (eg. Lucky tours, harty } \\
\text { tours, travco etc .) }\end{array}$ & 34 & 14.9 & 14.9 & & \\
\hline $\begin{array}{l}\text { Price comparison apps (e.g. } \\
\text { TripAdvisor, KAYAK etc.) }\end{array}$ & 114 & 50.0 & 50.0 & & \\
\hline Total & 228 & 100.0 & 100.0 & & \\
\hline
\end{tabular}

\begin{tabular}{|c|c|c|c|c|c|}
\hline \multicolumn{6}{|c|}{ If the trip has the same price where do you prefer to buy? } \\
\hline & Frequency & Percent & \begin{tabular}{|l} 
Valid \\
Percent
\end{tabular} & Mean & $\begin{array}{c}\text { Std. } \\
\text { Deviation }\end{array}$ \\
\hline Travel Agencies & 50 & 21.9 & 21.9 & \multirow{4}{*}{2.140} & \multirow{4}{*}{.7495} \\
\hline Internet in general & 96 & 42.1 & 42.1 & & \\
\hline Mobile Travel Applications & 82 & 36.0 & 36.0 & & \\
\hline Total & 228 & 100.0 & 100.0 & & \\
\hline \multicolumn{6}{|c|}{ I usually buy my flight ticket and book my hotel using } \\
\hline & Frequency & Percent & $\begin{array}{c}\text { Valid } \\
\text { Percent }\end{array}$ & Mean & $\begin{array}{c}\text { Std. } \\
\text { Deviation }\end{array}$ \\
\hline Kayak & 46 & 20.2 & 20.2 & \multirow{9}{*}{3.526} & \multirow{9}{*}{1.9877} \\
\hline sky scanner & 52 & 22.8 & 22.8 & & \\
\hline air BNB & 16 & 7.0 & 7.0 & & \\
\hline booking .com & 72 & 31.6 & 31.6 & & \\
\hline cheap flights & 22 & 9.6 & 9.6 & & \\
\hline Trivaco & 2 & .9 & .9 & & \\
\hline Yatra & 2 & 99 & 9 & & \\
\hline hostel bookers & 4 & 1.8 & 1.8 & & \\
\hline Others & 12 & 5.3 & 5.3 & & \\
\hline
\end{tabular}




\begin{tabular}{|c|c|c|c|c|c|c|}
\hline Total & & 228 & 100.0 & 100.0 & & \\
\hline \multicolumn{7}{|c|}{$\begin{array}{l}\text { Buying a trip using a Mobile Travel Application is the } \\
\text { most convenient way of shopping }\end{array}$} \\
\hline & & Frequency & Percent & $\begin{array}{c}\text { Valid } \\
\text { Percent }\end{array}$ & Mean & $\begin{array}{c}\text { Std. } \\
\text { Deviation }\end{array}$ \\
\hline Yes & & 182 & 79.8 & 79.8 & \multirow[t]{3}{*}{1.202} & \multirow[t]{3}{*}{.4022} \\
\hline No & & 46 & 20.2 & 20.2 & & \\
\hline Total & & 228 & 100.0 & 100.0 & & \\
\hline \multicolumn{7}{|c|}{$\begin{array}{c}\text { Buying trips using Mobile Travel Applications is less time consuming than buying them } \\
\text { traditionally from the travel agencies }\end{array}$} \\
\hline & Frequency & Percent & $\begin{array}{c}\text { Valid } \\
\text { Percent }\end{array}$ & Mean & \multicolumn{2}{|c|}{ Std. Deviation } \\
\hline Yes & 202 & 88.6 & 88.6 & \multirow[b]{3}{*}{1.114} & \multirow{3}{*}{\multicolumn{2}{|c|}{.3186}} \\
\hline No & 26 & 11.4 & 11.4 & & & \\
\hline Total & 228 & 100.0 & 100.0 & & & \\
\hline
\end{tabular}

\begin{tabular}{|c|c|c|c|c|c|}
\hline \multicolumn{6}{|c|}{ Mobile travel applications help me to make the right buying decision } \\
\hline & Frequency & Percent & $\begin{array}{c}\text { Valid } \\
\text { Percent }\end{array}$ & Mean & Std. Deviation \\
\hline Yes & 200 & 87.7 & 87.7 & \multirow{3}{*}{1.123} & \multirow{3}{*}{.3289} \\
\hline No & 28 & 12.3 & 12.3 & & \\
\hline Total & 228 & 100.0 & 100.0 & & \\
\hline
\end{tabular}

Results revealed that more than half of the sample book their hotels and flights via mobile applications $64 \%$ book hotels via mobile application and $55.3 \%$ | book flights via mobile applications $.37 .7 \%$ of the respondents have indicated that they book their flights through Online indirect and $4.4 \%$ book their flights through a booking agency . 62.71\% have booked their hotels through Online indirect and 5.26\% have booked their hotels through a booking agency, which means that travelers' attitude towards travel agencies has changed and they are not willing to get tourism services through them. Also, they have mentioned that the most used travel applications in booking a trip are the price comparison applications, $50 \%$ of users utilize them. Furthermore, $42.1 \%$ of the respondents choose to buy their trip through internet in general and $21.9 \%$ choose travel agencies which is an indicator that respondents prefer online to offline tools to select their trips. This should be put in to consideration when marketing a travel agencies offers. The most used travel applications are booking.com, sky scanner and kayak and other mobile travel applications include yelp, easy jet, ease my trip and make my trip .Customers also buy directly from hotel's websites or airline websites. $79.8 \%$ of respondents indicated that buying a trip using a Mobile Travel Application is the most convenient way of shopping and $88.6 \%$ responded 
that Mobile Travel Applications are less time consuming than buying them traditionally from the travel agencies Also, $87.7 \%$ indicated that travel applications help them make the right buying decision.

Table 4 : Usage of mobile travel application

\begin{tabular}{|c|c|c|c|c|c|}
\hline \multicolumn{6}{|c|}{ How often do you use your travel mobile applications on your smartphone/tablet? } \\
\hline & Frequency & Percent & Valid Percent & Mean & Std. Deviation \\
\hline Never & 50 & 21.9 & 21.9 & \multirow{6}{*}{2.377} & \multirow{6}{*}{.9790} \\
\hline Once a week & 66 & 28.9 & 28.9 & & \\
\hline Some days & 96 & 42.1 & 42.1 & & \\
\hline Once a day & 8 & 3.5 & 3.5 & & \\
\hline All day long & 8 & 3.5 & 3.5 & & \\
\hline Total & 228 & 100.0 & 100.0 & & \\
\hline \multicolumn{6}{|c|}{ Do you use your Mobile Travel Application when you are } \\
\hline Planning a trip & 186 & 81.6 & 81.6 & \multirow{5}{*}{1.254} & \multirow{5}{*}{.6056} \\
\hline Buying a trip & 30 & 13.2 & 13.2 & & \\
\hline On a trip & 8 & 3.5 & 3.5 & & \\
\hline After a trip & 4 & 1.8 & 1.8 & & \\
\hline Total & 228 & 100.0 & 100.0 & & \\
\hline \multicolumn{6}{|c|}{$\begin{array}{c}\text { What is the best service when using a Mobile Travel } \\
\text { Application? }\end{array}$} \\
\hline Flight Booking & 86 & 37.7 & 37.7 & \multirow{5}{*}{2.079} & \multirow{5}{*}{1.5715} \\
\hline Hotel Booking & 104 & 45.6 & 45.6 & & \\
\hline Car Rental Booking & 10 & 4.4 & 4.4 & & \\
\hline $\begin{array}{l}\text { General information } \\
\text { of the destination }\end{array}$ & 28 & 12.3 & 12.3 & & \\
\hline Total & 228 & 100.0 & 100.0 & & \\
\hline
\end{tabular}

The results in table ( 4 ) revealed that $42.1 \%$ of respondents use travel mobile applications on their smart phones for some days , $81.6 \%$ indicated that they use mobile travel application while they are planning a trip and 45.6 $\%$ showed that the best service provided when using mobile travel application is hotel booking followed by flight booking.

Table 5 : checking travel agencies website

\begin{tabular}{|c|c|c|c|c|c|}
\hline \multicolumn{7}{|c|}{ - Do you check travel agencies websites } \\
\hline & Frequency & Percent & Valid Percent & Mean & Std. Deviation \\
\hline Yes & 114 & 50.0 & 50.0 & \multirow{2}{*}{1.500} & .5011 \\
\hline no & 114 & 50.0 & 50.0 & & \\
\hline Total & 228 & 100.0 & 100.0 & & \\
\hline
\end{tabular}




\begin{tabular}{|c|c|c|c|c|c|}
\hline \multicolumn{6}{|c|}{ Do you view the offers of the travel agencies on face book mobile applications? } \\
\hline & Frequency & Percent & Valid Percent & Mean & Std. Deviation \\
\hline Yes & 116 & 50.9 & 50.9 & \multirow[t]{2}{*}{1.491} & \multirow[t]{2}{*}{.5010} \\
\hline no & 112 & 49.1 & 49.1 & & \\
\hline Total & 228 & 100.0 & 100.0 & & \\
\hline \multicolumn{6}{|c|}{ The prices of the travel agencies published on their websites are appropriate for a domestic trip } \\
\hline & Frequency & Percent & Valid Percent & Mean & Std. Deviation \\
\hline Yes & 156 & 68.4 & 68.4 & \multirow[t]{2}{*}{1.316} & \multirow[t]{2}{*}{.4659} \\
\hline no & 72 & 31.6 & 31.6 & & \\
\hline Total & 228 & 100.0 & 100.0 & & \\
\hline \multicolumn{6}{|c|}{$\begin{array}{l}\text { The prices of the travel agencies published on their websites are appropriate for an international } \\
\text { trip }\end{array}$} \\
\hline & Frequency & Percent & Valid Percent & Mean & Std. Deviation \\
\hline Yes & 82 & 36.0 & 36.0 & 1.360 & .4810 \\
\hline no & 146 & 64.0 & 64.0 & & \\
\hline Total & 228 & 100.0 & 100.0 & & \\
\hline
\end{tabular}

As shown in table (5) the agreement level of all respondents with all statements are positive which means $1.500,1.491,1.316$,and1.360 .However it is worth mentioning that these measures are insufficient, and misleading . Furthermore, the results shows, that half of the respondents only check travel agencies websites and view the offers of the travel agencies on facebook In addition $68.4 \%$ responded that the prices for a domestic trip published on travel agencies websites are appropriate however $64 \%$ said that the prices for an international trip published on their websites are not appropriate. They said that the offers of the travel agencies for international trips had often expired on their websites. The prices ae a lot higher than the prices online. online they get a better value for their money . Travel agencies also charge a much higher commission which reflects on the total price. The prices of travel agencies are very expensive for international trips but appropriate for domestic trips. Travel agencies also charge high commissions on sightseeing, ask tourists to pay in US dollars regardless of the currency of their countries, and the charge other hidden costs . 
Table 6: Customer loyalty to travel mobile applications

\begin{tabular}{|c|c|c|c|c|c|c|c|}
\hline Customer loyalty & $\begin{array}{l}\mathrm{S} \\
\mathrm{D} \\
\%\end{array}$ & $\begin{array}{l}\text { D } \\
\%\end{array}$ & $\begin{array}{l}\mathbf{N} \\
\%\end{array}$ & $\begin{array}{l}\text { A } \\
\%\end{array}$ & $\begin{array}{l}\text { SA } \\
\%\end{array}$ & Mean & $\begin{array}{c}\text { Std. } \\
\text { Deviation }\end{array}$ \\
\hline $\begin{array}{l}\text { I am very likely to use such } \\
\text { travel mobile applications }\end{array}$ & 0 & 5.3 & 21.9 & 46.5 & 26.4 & 3.939 & .8320 \\
\hline $\begin{array}{l}\text { I have a positive attitude } \\
\text { towards the application }\end{array}$ & 0 & 1.8 & 16.7 & 51.8 & 29.8 & 4.096 & .7267 \\
\hline $\begin{array}{l}\text { I would prefer such travel } \\
\text { applications over the travel } \\
\text { agencies }\end{array}$ & 0 & 8.8 & 26.3 & 43.0 & 21.9 & 3.781 & .8881 \\
\hline $\begin{array}{l}\text { I make my travel plans } \\
\text { through mobile applications }\end{array}$ & 0 & 8.8 & 20.2 & 44.7 & 26.3 & 3.886 & .8980 \\
\hline $\begin{array}{l}\text { I choose historical sites on } \\
\text { the basis of user comments } \\
\text { on Trip Advisor }\end{array}$ & 0 & 8.8 & 22.8 & 47.4 & 21.1 & 3.807 & .8692 \\
\hline $\begin{array}{l}\text { I travel cities more easily } \\
\text { with GPS }\end{array}$ & 0 & 4.4 & 7.9 & 43.0 & 44.7 & 4.281 & .7910 \\
\hline $\begin{array}{l}\text { I find cheap flights through } \\
\text { mobile applications more } \\
\text { than travel agencies }\end{array}$ & 0 & 4.4 & 21.9 & 43.9 & 29.8 & 3.991 & .8342 \\
\hline $\begin{array}{l}\text { I read user reviews before } \\
\text { making hotel reservations } \\
\text { through trip advisor }\end{array}$ & 0 & 3.5 & 22.8 & 47.4 & 26.3 & 3.965 & .7957 \\
\hline $\begin{array}{l}\text { I make a price comparison } \\
\text { among hotels using mobile } \\
\text { applications }\end{array}$ & 0 & 5.3 & 9.6 & 54.4 & 30.7 & 4.105 & .7782 \\
\hline $\begin{array}{l}\text { I prefer mobile applications } \\
\text { to find companies for } \\
\text { transfer from the airport }\end{array}$ & 0 & 12.3 & 28.1 & 38.6 & 21.1 & 3.684 & .9416 \\
\hline $\begin{array}{l}\text { I do not need a tour guide as } \\
\text { i can utilize mobile } \\
\text { applications instead. }\end{array}$ & 0 & 14.0 & 19.3 & 45.6 & 21.1 & 3.737 & .9485 \\
\hline $\begin{array}{l}\text { I find the places of } \\
\text { entertainment by using } \\
\text { mobile applications }\end{array}$ & 0 & 7.0 & 14.9 & 56.1 & 21.9 & 3.930 & .8044 \\
\hline $\begin{array}{l}\text { I choose restaurants } \\
\text { according to reviews and } \\
\text { comments on mobile } \\
\text { applications }\end{array}$ & 0 & 8.8 & 17.5 & 44.7 & 28.9 & 3.939 & .9031 \\
\hline $\begin{array}{l}\text { Mobile travel applications } \\
\text { have important impact on } \\
\text { the tourism }\end{array}$ & 0 & 1.8 & 8.8 & 52.6 & 36.8 & 4.246 & .6841 \\
\hline $\begin{array}{l}\text { I can rely on mobile travel } \\
\text { applications rather than } \\
\text { going to a travel agency }\end{array}$ & 0 & 8.8 & 12.3 & 50.9 & 28.1 & 3.982 & .8703 \\
\hline \multicolumn{6}{|l|}{ TOTAL $(\mathrm{N}=228)$} & 3.957 & 0.172041 \\
\hline
\end{tabular}


To understand Customer loyalty to travel mobile applications, the respondents have been asked to rate their level of agreement or disagreement as listed in table 6 . As shown above, most customers view online applications positively with mean ( 3.957 ) and standard deviation (0.172041). This means that most customers are loyal to travel mobile applications .

\section{Regression Analysis test}

Table 7: H1: Customer loyalty has a positive influence on buying

\section{behavior}

\section{Linear Regression}

\begin{tabular}{|c|c|c|c|c|c|c|c|c|c|}
\hline \multicolumn{10}{|c|}{ Model Summary ${ }^{b}$} \\
\hline \multirow[b]{2}{*}{ Model } & \multirow[b]{2}{*}{$\mathrm{R}$} & \multirow[b]{2}{*}{\begin{tabular}{c|}
$\mathrm{R}$ \\
Square
\end{tabular}} & \multirow[b]{2}{*}{$\begin{array}{c}\text { Adjusted R } \\
\text { Square }\end{array}$} & \multirow[b]{2}{*}{$\begin{array}{l}\text { Std. Error of the } \\
\text { Estimate }\end{array}$} & \multicolumn{5}{|c|}{ Change Statistics } \\
\hline & & & & & $\begin{array}{l}\text { R Square } \\
\text { Change }\end{array}$ & $\begin{array}{c}\mathrm{F} \\
\text { Change }\end{array}$ & $\mathrm{dfl}$ & $\mathrm{df} 2$ & $\begin{array}{l}\text { Sig. F } \\
\text { Change }\end{array}$ \\
\hline 1 & $.668^{\mathrm{a}}$ & .447 & .444 & \begin{tabular}{|l|l|}
7.80259 \\
\end{tabular} & .447 & 182.359 & 1 & 226 & .000 \\
\hline \multicolumn{10}{|c|}{ a. Dependent Variable: Customer loyalty } \\
\hline$\overline{\text { b. } P}$ & 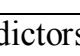 & onst & ant), Buyıng & chavior & & & & & \\
\hline
\end{tabular}

According to the results in the previous table, which measures the relationship between customer loyalty and busying behavior, the overall regression model fit statistically. The " $\mathrm{R}$ " column represents the value of $\mathrm{R}$, the multiple correlation coefficient. $\mathrm{R}$ can be considered to be one measure of the quality of the prediction of the dependent variable.

A value of 0.668 indicates a good level of prediction. The "R Square" column represents the R2 value. The value of 0.447 shows that the independent variables explain $44.7 \%$ of the variance of the dependent variable and it is highly significant $(\mathrm{p}<0.001)$

\begin{tabular}{l|l|c|c|c|c|c|}
\hline \multicolumn{7}{|l|}{ ANOVA $^{\mathbf{a}}$} \\
\hline \multirow{2}{*}{ Model } & Sum of Squares & Df & Mean Square & F & Sig. \\
\cline { 2 - 7 } & Regression & 11102.083 & 1 & 11102.083 & 182.359 & $\mathbf{. 0 0 0}^{\mathbf{b}}$ \\
\cline { 2 - 7 } & Residual & 13758.969 & 226 & 60.880 & & \\
\cline { 2 - 7 } & Total & 24861.053 & 227 & & & \\
\hline \multicolumn{2}{l}{ a. Dependent Variable: Customer loyalty } \\
b. Predictors: (Constant), Buying behavior
\end{tabular}


The $F$-ratio in the ANOVA table (2) tests whether the overall regression model is a good fit for the data. The table shows that the independent variables statistically significantly predict the dependent variable, $\mathrm{F}(1,226)=$ $182.359, \mathrm{p}<.0005$ (the regression model is a good fit of the data). Thus the results indicate that there is a linear relationship between the variables in the model.

\section{Coefficients a}

\begin{tabular}{|c|c|c|c|c|c|c|c|c|c|c|c|c|}
\hline & \multicolumn{3}{|c|}{$\begin{array}{c}\text { Unstandardized Standardized } \\
\text { Coefficients Coefficients }\end{array}$} & \multirow{2}{*}{$\mathrm{T}$} & \multirow{2}{*}{ Sig. } & \multicolumn{2}{|c|}{$\begin{array}{l}95.0 \% \\
\text { Confidence } \\
\text { Interval for B }\end{array}$} & \multicolumn{3}{|c|}{ Correlations } & \multicolumn{2}{|c|}{$\begin{array}{l}\text { Collinearity } \\
\text { Statistics }\end{array}$} \\
\hline & B & $\begin{array}{l}\text { Std. } \\
\text { Error }\end{array}$ & Beta & & & $\begin{array}{l}\text { Lower } \\
\text { Bound }\end{array}$ & $\begin{array}{l}\text { Upper } \\
\text { Bound }\end{array}$ & $\begin{array}{l}\text { Zero- } \\
\text { order }\end{array}$ & Partial & Part & Tolerance & VIF \\
\hline 1 (Constant & 22.357 & 2.789 & & 8.016 & .000 & 16.861 & 27.853 & & & & & \\
\hline $\begin{array}{l}\text { Buying } \\
\text { behavior }\end{array}$ & 1.981 & 147 & .668 & 13.504 & .000 & 1.692 & 2.270 & .668 & .668 & .668 & 1.000 & 1.000 \\
\hline \multicolumn{13}{|c|}{ a. Dependent Variable: Custom } \\
\hline
\end{tabular}

The model shows that the linear regression analysis estimates the linear regression function to be $\mathrm{y}=22.357+1.981 * \mathrm{x}$. This means that an increase in one unit of $x$ leads to an increase of the $y$ unit by 1.981. It is shown from the "Sig." column that all independent variable coefficients are statistically significantly different from 0 (zero). Also, there is a medium positive correlation between the two variables (customer loyalty and buying behavior) were $(r=0.668)$. Which proves the first hypothesis: the Customer loyalty has a positive influence on buying behavior 
Table 8 :H2: usage of mobile travel application has a positive influence on travel agencies website

\begin{tabular}{|c|c|c|c|c|c|c|c|c|c|c|}
\hline \multicolumn{11}{|c|}{ Model Summaryb } \\
\hline \multirow[b]{2}{*}{ Model } & \multirow[b]{2}{*}{ R } & \multirow[b]{2}{*}{$\begin{array}{l}\mathrm{R} \\
\text { Square }\end{array}$} & \multirow[b]{2}{*}{$\begin{array}{l}\text { Adjusted R } \\
\text { Square }\end{array}$} & \multirow[b]{2}{*}{$\begin{array}{l}\text { Std. Error of } \\
\text { the Estimate }\end{array}$} & \multicolumn{5}{|c|}{ Change Statistics } & \multirow[b]{2}{*}{$\begin{array}{l}\text { Durbin- } \\
\text { Watson }\end{array}$} \\
\hline & & & & & $\begin{array}{l}\text { R Square } \\
\text { Change }\end{array}$ & F Change & e df1 & $\mathrm{df} 2$ & $\begin{array}{l}\text { Sig. F } \\
\text { Change }\end{array}$ & \\
\hline 1 & .602 & $2 \mathrm{a} .363$ & .360 & 1.63750 & .363 & 128.625 & 1 & 226 & .000 & .311 \\
\hline
\end{tabular}

The researcher used the regression analysis to measure the relationship between usage of mobile travel application and travel agencies websites. According to Table ( 8 ), the overall regression model fits statistically. The " $\mathrm{R}$ " column represents the value of $\mathrm{R}$, the multiple correlation coefficient. $\mathrm{R}$ can be considered one measure of the quality of the prediction of the dependent variable.

A value of .602 indicates a good level of prediction. The "R Square" column represents the R2 value. It shows from the value of .363 that the independent variables explain $36.3 \%$ of the variability of the dependent variable and it is highly significant $(\mathrm{p}<0.001)$

\begin{tabular}{|c|c|c|c|c|c|c|}
\hline \multicolumn{7}{|c|}{ ANOVAa } \\
\hline \multicolumn{2}{|c|}{ Model } & Sum of Squares & $\mathrm{df}$ & Mean Square & $\mathrm{F}$ & Sig. \\
\hline \multirow[t]{3}{*}{1} & Regression & 344.896 & 1 & 344.896 & 128.625 & $.000^{\mathrm{b}}$ \\
\hline & Residual & 605.999 & 226 & 2.681 & & \\
\hline & Total & 950.895 & 227 & & & \\
\hline \multicolumn{7}{|c|}{ a. Predictors: (Constant), usage of mobile travel application } \\
\hline \multicolumn{7}{|c|}{ b. Dependent Variable: travel agencies website } \\
\hline
\end{tabular}

The $F$-ratio in the ANOVA table tests whether the overall regression model is a good fit for the data. The table shows that the independent variables statistically significantly predict the dependent variable, $\mathrm{F}(1,226)=128.625$, $\mathrm{p}<.0005$ (the regression model is a good fit of the data), Thus the results indicate that there is a linear relationship between the variables in the model 


\begin{tabular}{|c|c|c|c|c|c|c|c|c|c|c|c|}
\hline \multicolumn{12}{|c|}{ Coefficientsa } \\
\hline \multirow[b]{3}{*}{ del } & \multirow{2}{*}{\multicolumn{2}{|c|}{$\begin{array}{c}\text { Unstandardized } \\
\text { Coefficients }\end{array}$}} & \multirow{3}{*}{\begin{tabular}{|c|} 
Standardized \\
Coefficients \\
Beta
\end{tabular}} & \multirow[b]{3}{*}{$\mathrm{t}$} & \multirow[b]{3}{*}{ Sig. } & \multirow[t]{2}{*}{\begin{tabular}{|c|}
$95.0 \%$ \\
Confidence \\
Interval for B
\end{tabular}} & \multirow{2}{*}{\multicolumn{3}{|c|}{ Correlations }} & \multirow{2}{*}{\multicolumn{2}{|c|}{$\begin{array}{c}\text { Collinearity } \\
\text { Statistics }\end{array}$}} \\
\hline & & & & & & & & & & & \\
\hline & $\mathrm{B}$ & \begin{tabular}{|c|} 
Std. \\
Error \\
\end{tabular} & & & & \begin{tabular}{|l|l|} 
Lower & Upper \\
Bound & Bound \\
\end{tabular} & $\begin{array}{l}\text { Zero- } \\
\text { order }\end{array}$ & Partial & 1 Part & Tolerance & VIF \\
\hline 1 (Constant) & 8.681 & .282 & & 30.752 & .000 & 8.89410 .313 & & & & & \\
\hline offers & -.528 & .0 .47 & -.602 & -11.341 & .000 & \begin{tabular}{|l|l|}
-.806 & -.568 \\
\end{tabular} & -.602 & -.602 & -.602 & 1.000 & 1.000 \\
\hline \multicolumn{12}{|c|}{ a. Predictors: (Constant), usage of mobile travel application } \\
\hline b. Dependen & nt Varial & le: trave & el agencies we & ebsite & & & & & & & \\
\hline
\end{tabular}

The model shows that the linear regression analysis estimates the linear regression function to be $\mathrm{y}=8.681+(-.528) * \mathrm{x}$. This means that any increase in one unit of $\mathrm{x}$ leads to a decrease in a y unit by (-.528). It is shown from the "Sig." column that all independent variable coefficients are statistically significantly different from 0 (zero). Consequently, there is a medium negative correlation between the two variables (usage of mobile travel application and travel agencies website) were $(\mathrm{r}=-0.602)$. The results revealed that the second hypothesis was correct. The use of mobile travel applications has a positive influence on travel websites.

\section{Results of correlation tests}

Table $9: \mathrm{H3}$ : There is a relationship between Demographic and customer loyalty

\begin{tabular}{|c|c|c|c|c|c|}
\hline \multicolumn{6}{|c|}{ Correlations } \\
\hline & & Gender & Age & Education & Loyalty \\
\hline \multirow[t]{3}{*}{ Gender } & Pearson Correlation & 1 & $.712 * *$ & $-.689 * *$ & .045 \\
\hline & Sig. (2-tailed) & & .000 & .000 & .495 \\
\hline & $\mathrm{N}$ & 228 & 228 & 228 & 228 \\
\hline \multirow[t]{3}{*}{ Age } & Pearson Correlation & $.712 * *$ & 1 & $-.521 * *$ & $-.313 * *$ \\
\hline & Sig. (2-tailed) & .000 & & .000 & .000 \\
\hline & $\mathrm{N}$ & 228 & 228 & 228 & 228 \\
\hline \multirow[t]{3}{*}{ Education } & Pearson Correlation & $-.689 * *$ & $-.521 * *$ & 1 & $-.302 * *$ \\
\hline & Sig. (2-tailed) & .000 & .000 & & .000 \\
\hline & $\mathrm{N}$ & 228 & 228 & 228 & 228 \\
\hline \multirow[t]{3}{*}{ Loyality } & Pearson Correlation & .045 & $-.313^{* *}$ & $-.302 * *$ & 1 \\
\hline & Sig. (2-tailed) & .495 & .000 & .000 & \\
\hline & $\mathrm{N}$ & 228 & 228 & 228 & 228 \\
\hline
\end{tabular}

Pearson's bivariate correlation coefficient shows a medium negative linear relationship between customer loyalty, age and education scores $(r=-.313$, $.302)$ that is significantly different from zero $(p<0.001)$. However, Pearson's 
bivariate correlation coefficient shows a non-significant linear relationship between customer loyalty, and gender $(\mathrm{r}=0.45)$. The findings confirm the third hypothesis, which is that there is a relationship between demographic characteristics and customer loyalty.

Based on the previous results, Table 10 shows the results of hypotheses testing.

Table 10

The Results of Hypothesis Testing

\begin{tabular}{|c|l|c|}
\hline$!$ & \multicolumn{1}{|c|}{ Hypothesis } & Results \\
\hline H1 & $\begin{array}{l}\text { Customer loyalty has a positive influence on buying } \\
\text { behavior. }\end{array}$ & accepted \\
\hline H2 & $\begin{array}{l}\text { Usage of mobile travel application has a positive influence } \\
\text { on travel agencies website }\end{array}$ & accepted \\
\hline H3 & $\begin{array}{l}\text { There is a relationship between Demographic and customer } \\
\text { loyalty }\end{array}$ & accepted \\
\hline
\end{tabular}

\section{Conclusion}

The study measures the impact of mobile travel applications on tourism industries. The main aim of this study is to determine the users' opinions of using Mobile travel Applications, evaluate their view of travel agencies websites, and Identify the relationship between demographic and customer loyalty. The study use multiple regression to predict the how Customer loyalty influences buying behavior. These variables statistically significantly predicted, $\mathrm{F}(1,226)=182.359, \mathrm{p}<.0005, \mathrm{R} 2=.447$, medium positive correlation between the two variables were $(r=0.668)$. However, the linear regression analysis estimates the linear regression function to be $y=22.357+$ $1.981^{*} \mathrm{x}$. This means that an increase in one unit of $\mathrm{x}$ leads to an increase in a unit $y$ unit by 1.981 . All variables added statistically significantly to the prediction, $\mathrm{p}<.05$. Furthermore, A multiple regression was used to predict the influence between Customer loyalty and buying behavior. These variables statistically significantly predicted that, $\mathrm{F}(1,226)=128.625, \mathrm{p}<.0005, \mathrm{R} 2=$ .363 , medium negative correlation between the two variables were $(\mathrm{r}=$ $0.602)$. The linear regression analysis estimates the linear regression function to be $\mathrm{y}=8.681+(-.528)^{*} \mathrm{x}$. This means that the increase in one unit of $\mathrm{x}$ leads to a decrease of unit y by (-.528). All variables added the statistically significantly to the prediction, $\mathrm{p}<.05$. In addition, there is a negative medium relationship between customer loyalty and the two demographic 
variables (age and education) However, the results indicated that there is no relationship between gender and customer loyalty.

\section{Recommendations}

- By using mobile apps, travel agencies can increase the number of their customers through retaining old clients and attracting new ones

- Through mobile travel applications, travel agencies can strengthen their market image establish a strong relationship with their customers, which will lead to customer loyalty .

- Traditional travel agencies have to acquire new electronic commerce technologies and enter the online travel segment by adopting strategies based on new technologies in order to survive in the new competitive environment (Chircu,Kauffman, 1999 ; Lin et al., 2009)

- changing the travel agencies website to have a mobile friendly layout.

- Travel agents should register in viator debuts as it is a booking platform for travel agents .It offers a lower prices for an identical activity and if the agent or customer finds a lower price, viator will refund the difference and the process is free (Skift, 2017)

\section{8-Research Limitation and Future Researches}

The data was mostly collected from the bachelor's degree, master's degree and $\mathrm{PhD}$ degree holders who represent mostly Gen $\mathrm{Y}$ (their ages vary between 21-40). They are known as "incredibly sophisticated, technology wise, immune to most traditional marketing and sales pitches as they not only grew up with it all, they've seen it all and been exposed to it all since early childhood"'( Wjs, 2018 ). Members of this generation usually have money, they are well-educated and they usually use mobile internet and applications for work .So, it is easier for them to use smart phones to purchase products over the internet. Very few people from older age categories have responded to the questionnaire, probably because they do not use the internet for shopping purposes.

The recent study focused on studying the impact of mobile travel applications on tourism, whereas future studies could focus on mobile friendly hotels' websites in Egypt and the impact of changing travel agencies websites to mobile friendly websites. In the future I can conduct a similar survey to collect data from different demographic profiles such as generation $\mathrm{x}$ and baby boomers to determine whether there is any difference between studies of various demographic profiles. 


\section{References}

1. Alshattnawi ,S. 2013. Building Mobile Tourist Guide Applications using Different Development Mobile Platforms. International Journal of Advanced Science and Technology . Jordan, Irbid, Yarmouk University, Faculty of Information Technology and Computer Science , 54, ,pp.1-10

2. Bicen, H. And Sadikoglu, S. (2015) Determination Of The Opinions Of Students On Tourism Impact Using Mobile Applications. 3rd Global Conference On Business, Economics, Management And Tourism, 26-28 November.Elsevier.pp.270-274

3. Buhalis, D. and Costa, C. 2006. Tourism Business Frontiers. OX: Butterworth-Heinemann : Elsevier. pp.13

4. Business insider .2013. The Mobile Tourist: How Smartphones Are Shaking Up The Travel Market. http://www.businessinsider.com/the-mobile-tourist-howsmartphones-are-shaking-up-the-travel-market-2013-2[Accessed 7 October 2017]

5. Chircu, A.M. and Kauffman, R.J., (1999), Strategies for Internet Middlemen in

the Intermediation/ Disintermediation/Re-intermediation Cycle, Electronic Markets- The International Journal of Electronic Commerce and Business Media (9:2), pp. 109-117

6. Chulmo, k., Seunghun, S., Keehun, K ; Chulwon, K., and Namho,C. 2013. Smart Tourism of the Korea: A Case Study. PACIS 2013 Proceedings. 138.

https://pdfs.semanticscholar.org/8074/446789aca29337218ffa0b6e82 2b3280ab00.pdf[Accessed 27 November 2017]

7. Fiedler, J. 2013 .An Airline's Mobile Travel Guide Application Effects on Customer Loyalty. Unpublished Master Thesis .International Marketing and Management. Copenhagen Business School .pp.42,60-63

8. Good worklabs. 2015 .How mobile app benefits travel and tourism industry https://www.goodworklabs.com/how-mobile-app-benefitstravel-and-tourism-industry/[Accessed 25 October 2017]

9. Grieve, C., Bendon, T., Hundson, S. 2010. Our Mobile Future: How Smartphones Will Transform Visiting Experiences. London: Horizon Digital Economy Research.pp.1

10. Moritz,C. 2015.Mobile Application Development in the Tourism Industry Industry and its Impact on Site Travel Behavior. Bachelor Thesis for obtaining the degree Bachelor of Business Administration 
Tourism and Hospitality Management .modul Vienna university. $\mathrm{pp}, 58$.

11. Jieun,K. Yongtae,P., K, Chulhyun. and L, Hakyeon .2014. Mobile application service networks: Apple's AppStore, Service business, 8( 1) .pp. $1-27$,

12. Kenteris, M., Gavalas, D., and Economou, D. 2007. An innovative mobile electronic tourist guide application. Pers Ubiquit Comput, 13, pp.103-118.

13. Langelund, S.2007. Mobile Travel. Tourism and Hospitality Research: Special Issue: Innovation for Sustainable Tourism, 7(3-4), pp.284-286.

14. Lin, D., Zhou, Z. and Guo, X. 2009. A Study of the Website Performance of Travel Agencies Based on the EMICA Model. J. Service Science \& Management, 3. pp. 181-185

15. Mamaghani, F. 2009. Impact of E-commerce on Travel and Tourism: An Historical Analysis. International Journal of Management, 26(3), pp.365-375.

16. Pallant, J. 2005. SPSS Survival Manual: A Step by Step Guide to Data Analysis Using SPSS Version 12. Chicago, IL: Open University Press.

17. Parro, L (2013). Smart Traveling With Smartphones - Or Is It? Study How Usable Are Mobile Travel Applications. Bachelor's Thesis Degree Program In Hospitality Management Production In Management Of Services.Turku Universty Of Applied Sciences .pp. 41-43

18. Skift,2017 -https://skift.com/2017/10/03/tripadvisor-tours-andactivities-brand-viator-debuts-a-booking-tool-for-travel-agents/ [Accessed 8 October 2017]

19. Tourism e kit . Mobile Technology For Tourism http://www.tourism.vic.gov.au/images/stories/ATDW_EKIT/tourism_ 20. e_kit_v7_50.pdf[Accessed 9 November 2017]

ang, D., Xiang, Z. and Fesenmaier D.R., 2014. Smartphone Use in Everyday Life and Travel. SAGE Publications. Journal of Travel Research https://www.researchgate.net/profile/Zheng_Xiang5/publication/2631 60992_Smartphone_Use_in_Everyday_Life_and_Travel/links $/ 545 \mathrm{f} 7 \mathrm{~b}$ 620cf295b56161c4a9/Smartphone-Use-in-Everyday-Life-andTravel.pdf [Accessed 18 April2015] 
21. Wtm. 2017

.http://www.wtmlondon.com/RXUK/RXUK_WTMLondon/2015/doc uments/WTM-Global-Trends-2014.pdf, [Accessed 2 December 2017]

22. WJS,2018 http://socialmarketing.org/archives/generations-xy-z-andthe-others/[Accessed 1 April 2018]

23. Yoo, K.H., and Gretzel, U. 2012. Use and Creation of Social Media by Travelers. In Sigala, M., Christou, E., \& Gretzel, U., Social Media in Travel, Tourism and Hospitality : theory, practice and cases . United Kingdom. Ashgate. Pp.189-206.

24. Yovchevaa,Z. Buhalisb,D.and Gatzidis,G. 2012. Overview of Smartphone Augmented Reality Applications for Tourism. e-Review of Tourism Research (eRTR), 10 (2) pp.63-66 